\title{
Renal denervation after the symplicity HTN-3 trial
}

\author{
Ewa Warchoł-Celińska1, Andrzej Januszewicz ${ }^{1}$, Aleksander Prejbisz ${ }^{1}$, Jacek Kądziela² \\ ${ }^{1}$ Department of Hypertension, Institute of Cardiology, Warsaw, Poland \\ ${ }^{2}$ Department of Interventional Cardiology and Angiology, Institute of Cardiology, Warsaw, Poland
}

Postep Kardiol Inter 2014; 10, 2 (36): 75-77

DOI: 10.5114/pwki.2014.43509

\section{Introduction}

Renal denervation (RDN) by radiofrequency ablation is a minimally invasive catheter-based technique targeting the renal sympathetic nerves which has emerged as a promising therapy for resistant hypertension since the initial Symplicity HTN-1 study was published in 2009 [1]. At the time, the role of sympathetic renal nerves in the development and course of hypertension had already been proven [2, 3] and the safety and efficacy of renal nerve ablation had been confirmed in animals models [4]. The "proof of concept" Symplicity HTN-1 trial demonstrated the safety and initial efficacy of the procedure in humans and led to the design of the first randomized trial, Symplicity HTN-2 [5]. Both trials revealed an impressive blood pressure-lowering effect and the safety of RDN $[1,5]$. The 36-month long-term follow-up of the Symplicity HTN-1 study confirmed a sustained blood pressure (BP) reduction of 33/19 $\mathrm{mm} \mathrm{Hg}$ [6]. These promising results were also confirmed later in many registries reporting on the effects of RDN on office and ambulatory BP [7]. Also the early results of the Global Symplicity Registry (GSR) - an open label, multi-indication registry, currently being conducted worldwide to evaluate the safety and efficacy of RDN with the Symplicity RDN System - support the view that RDN is an effective and safe procedure in reducing $B P$.

The Symplicity Trials and later reports led to great enthusiasm in the medical community and resulted in a consensus statement on RDN of European experts published in the "European Heart Journal" [7] followed by the position statement of the European Society of Hypertension (ESH) [8] and current ESH/ESC Guidelines for the management of arterial hypertension [9]. According to the ESH/ESC Guidelines, renal denervation may be considered as a possible treatment for patients with resistant hypertension meeting the restricted criteria for the procedure. Knowing the limitations of previous trials (including small sample sizes, limited assessment of ambulatory BP, lack of blinding, and lack of sham procedure as a control) all above-mentioned documents underlined however the necessity of a larger and properly designed, blinded trial with a masked procedure to determine the role of RDN.

\section{Symplicity HTN-3}

The Symplicity HTN-3 trial is the first blinded, randomized, sham-procedure controlled trial of RDN for treatment-resistant hypertension [10]. In March 2014 the highly surprising results of this eagerly awaited trial were presented at the American College of Cardiology. The Symplicity HTN-3 trial included patients with a systolic BP (SBP) $\geq 160 \mathrm{~mm} \mathrm{Hg}$ despite the concurrent use of three antihypertensive drugs (including a diuretic) at maximally tolerated dosages. From 1441 patients assessed for eligibility, 535 patients (37\%) from 88 medical centers in the USA were enrolled. Two weeks after initial screening, a confirmatory screening visit was conducted during which SBP $\geq 160 \mathrm{~mm} \mathrm{Hg}$ was confirmed, adherence to the therapy according to the patient's diary was documented and ambulatory BP monitoring was performed to confirm the hypertension resistance (daytime SBP average $\geq 135 \mathrm{~mm} \mathrm{Hg}$ ). Patients included in the study were randomly assigned in a $2: 1$ ratio to renal nerve ablation using the Symplicity Flex Catheter (Medtronic, MN, USA) or to renal angiography only (sham control). Both patients and BP assessors were unaware of the study group allocation. Changes of antihypertensive regimen were not allowed during the 6-month follow-up period unless they were clinically necessary. The primary efficacy endpoint was the change in office SBP at 6 months and the secondary efficacy endpoint was the change in mean 24-hour ambulatory SBP. The primary safety endpoint was a com-

Corresponding author:

Ewa Warchoł-Celińska MD, Department of Hypertension, Institute of Cardiology, 42 Alpejska St, 04-628 Warsaw, Poland, phone: +48 606529 045, e-mail: ewarchol@ikard.pl

Received: 2.06.2014, accepted: 2.06.2014. 
posite of major adverse events, and renal artery or other vascular complications within 6 months after treatment. As the primary safety end point was met, renal nerve ablation was confirmed to be a safe procedure with no major adverse events. Disappointingly however, RDN did not reach the primary efficacy endpoint of reduction in office $\mathrm{SBP}$, or the secondary efficacy endpoint of decrease in 24-hour ambulatory BP levels. In spite of a statistically significant decrease of BP at 6 months compared with baseline of $14.1 \mathrm{~mm} \mathrm{Hg}$ for RDN and $11.7 \mathrm{~mm} \mathrm{Hg}$ for the sham treatment group, the difference of $-2.29 \mathrm{~mm} \mathrm{Hg}$ in office SBP between the two groups was not significant $(p=0.26)$. Similarly, in both groups significant decreases in 24-hour ambulatory SBP levels at 6 months as compared to baseline were noted, whereas the difference between the two arms of $-1.96 \mathrm{~mm} \mathrm{Hg}$ remained non-significant $(p=0.98)$. In contradiction to most published data on RDN, in the Symplicity HTN-3 study renal nerve ablation appeared to have no significant effect on office or 24-hour ambulatory SBP levels as compared to the control group.

\section{Commentary}

Although the surprising results of the Symplicity HTN-3 trial questioned the future of renal nerve ablation for resistant hypertension, a critical view is needed to better understand the discrepancy between the previously known data on RDN and the new findings of Symplicity HTN-3. There are a number of possible explanations why Symplicity HTN-3 did not confirm the results of previous published registries and randomized trials.

The first concerns are associated with study design. Symplicity HTN-3 is the first blinded trial for RDN which included a sham-control group. The lack of a control group in previous studies might have led to a false impression of RDN treatment efficacy. In those non-randomized trials, the regression to mean phenomenon as well as the effect of trial participation (due to good care and a high degree of adherence resulting from close follow-up) may have positively affected the treatment efficacy. In the randomized trial Symplicity HTN-2 including a control group, a lack of blinding may have led to both patients' and assessors' bias in favor of a new treatment which is expected to be highly effective. Sham procedures are analogous to the use of a placebo in pharmaceutical studies and their implementation may result in the placebo effect, as was clearly observed in Symplicity HTN-3 [10]. The significant decreases of office and ambulatory BP at 6 months compared to baseline were noted not only in the RDN, but also in the sham-control group. If the placebo effect declines with time, the relatively short follow-up of 6 months might be too short to ascertain the primary end point. As the patients included in the study will be followed for up to 5 years, the future Symplicity HTN-3 results may bring findings in favor of RDN efficacy [10].
Nevertheless, it must also be noted that previous studies revealed twice as large an effect on BP lowering already at 6 months (sustained through 3 years) after RDN as compared to the corresponding group in Symplicity HTN-3. There are several possibilities why the relatively small decrease of BP levels was observed in Symplicity HTN-3. Possible explanations can be divided into those concerning issues associated with the procedure itself and those concerning enrolled patients' characteristics. The 535 patients included in the Symplicity HTN-3 study were recruited in 88 centers and overall 111 operators performed 364 renal denervation procedures. It has to be noted that renal nerve ablation is not approved in the USA and no roll-in phase was applied; therefore the operators were rather inexperienced and each of them performed a small number of around three procedures. This fact may have influenced the level of effectiveness and completeness of procedures performed in the study. In addition, there is no test available allowing assessment of proper wall contact and effective destruction of renal nerves during or after the procedure [11].

The population of previous studies consisted mainly of Caucasians recruited in Europe and Australia. A quarter of the patients enrolled in the Symplicity HTN-3 trial, however, were Afro-Americans and a detailed analysis suggested that this subgroup of patients responds better to sham than to RDN. What is also interesting is the fact that Afro-Americans allocated to the sham-control group had a more pronounced decline in office SBP as compared to the Caucasian and Asian patients in the sham arm [11]. The standard deviation of the change in office systolic BP from baseline confirmed a wide variability in response to RDN. This may suggest that this procedure could be more efficacious in the selected group of patients [12]. In Symplicity HTN-3 a significant difference between the change in office SBP was observed in patients who were not Afro-Americans, in younger patients ( $<65$ years) and in the subgroup of patients with preserved renal function $\left(\geq 60 \mathrm{ml} / \mathrm{min} / 1.73 \mathrm{~m}^{2}\right.$ ) [10]. The Symplicity HTN-3 population also differed from the population included in the previous studies in respect of pharmacological regimen. The percentage of direct-acting vasodilators was twice as high as in previous studies. Because of numerous side effects, this group of medications is associated with a low adherence rate, which may influence the high variability of office and ambulatory BP readings resulting from variation in adherence to the therapy with this group of medications. What is more, vasodilators appeared to be a predictor of non-response to RDN [11]. The percentage of aldosterone antagonists was also higher than in previous studies, which may have facilitated the decrease in BP levels in the control group [12].

Undoubtedly, the announcement of the Symplicity HTN-3 results have begun a new chapter in the field of renal nerve ablation for resistant hypertension. These unexpected results have caused much confusion, but also 
have brought new, challenging questions for the possible future use of RDN in the field of hypertension. Symplicity HTN-3 confirmed the safety of RDN, but also indicated possible groups of responders and non-responders to the procedure and underlined the necessity of developing reliable and practical tools to determine the effectiveness of renal nerve ablation. Although after having released the results of the Symplicity HTN-3 trial, Medtronic, Inc. suspended enrollment in their trials, the company decided to continue the Global Symplicity Registry and renal denervation studies evaluating other non-hypertension indications including hypertension coexisting with metabolic abnormalities and/or sleep apnea, chronic kidney disease, heart failure and arrhythmias. At the same time, beside Medtronic's Symplicity System, there are also other renal denervation systems available and they are successfully used in clinical trials [13, 14]. We cannot forget that renal denervation still has a place in the current ESH/ESC Guidelines for the management of arterial hypertension with the highly restricted criteria for this procedure [9]. Therefore, according to the ESH statement on Symplicity HTN-3 results: “...the reaction to the negative results of the Symplicity HTN-3 study should not be to abandon the renal denervation approach, but to perform further studies of high scientific calibre that could provide further evidence on its overall position in the treatment of resistant hypertension, determine whether the blood pressure effects are limited to some patients' subgroups (and clarify their characteristics), and see whether and to what extent their blood pressure reductions translate into cardiovascular and renal protection".

\section{References}

1. Krum $H$, Schlaich $M$, Whitbourn $R$, et al. Catheter-based renal sympathetic denervation for resistant hypertension: a multicentre safety and proof-of-principle cohort study. Lancet 2009; 373: 1275-81.

2. Kirchheim H, Ehmke H, Persson P. Sympathetic modulation of renal hemodynamics, renin release and sodium excretion. Klin Wochenschr 1989; 67: 858-64.

3. Kon V. Neural control of renal circulation. Miner Electrolyte Metab 1989; 15: 33-43.

4. Kadziela J, Florczak E, Januszewicz A, Witkowski A. Denerwacja nerek - nowa metoda leczenia opornego nadciśnienia tętniczego. Postep Kardiol Inter 2010; 6: 173-6.

5. Symplicity HTN-2 Investigators, Esler MD, Krum H, Sobotka PA, et al. Renal sympathetic denervation in patients with treatment-resistant hypertension (The Symplicity HTN-2 Trial): a randomised controlled trial. Lancet 2010; 376: 1903-9.

6. Krum H, Schlaich M, Sobotka PA, et al. TCT-12 long-termfollow-up of catheter-based renal denervation for resistant hypertension confirms durable blood pressure reduction. J Am Coll Cardiol 2012; 60: doi: 10.1016/j.jacc.2012.08.017.

7. Mahfoud F, Luscher TF, Andersson B, et al. Expert consensus document from the European Society of Cardiology on catheter-based renal denervation. Eur Heart J 2013; 34: 2149-57.
8. Schmieder RE, Redon J, Grassi G, et al. ESH position paper: renal denervation - an interventional therapy of resistant hypertension. J Hypertens 2012; 30: 837-41.

9. Mancia G, Fagard R, Narkiewicz K, et al. 2013 Guidelines for the Management of Arterial Hypertension: The Task Force for the Management of Arterial Hypertension of the European Society of Hypertension (ESH) and of the European Society of Cardiology (ESC). Eur Heart J 2013; 34: 2159-219.

10. Bhatt DL, Kandzari DE, O'Neill WW, et al. (SYMPLICITYHTN-3 Investigators). A controlled trial of renal denervation for resistant hypertension. N Engl J Med 2014; 370: 1393-401.

11. Luscher TF, Mahfoud F. Renal nerve ablation after SYMPLICITYHTN-3: confused at the higher level? Eur Heart J 2014 May 14. pii: ehu195 [Epub ahead of print].

12. Messerli FH, Bangalore S. Renal denervation for resistant hypertension? N Engl J Med 2014; 370: 1454-7.

13. Worthley SG, Tsioufis CP, Worthley MI. Safety and efficacy of a multi-electrode renal sympathetic denervation system in resistant hypertension: the EnligHTN I trial. Eur Heart J 2013; 34: 2132-40.

14. Jin Y, Jacobs L, Baelen M, et al. (Investigator-steered project on intravascular renal denervation for management of drug-resistant hypertension (INSPiRED) investigators). Rationale and design of the Investigator-Steered Project on intravascular Renal Denervation for Management of Drug-Resistant Hypertension (INSPiRED) trial. Blood Press 2014; 23: 138-46. 